\title{
Engajamento no Trabalho: Características Psicométricas e Invariância Temporal do UWES
}

\author{
Paulo Cesar Porto-Martins ${ }^{1}$ (ID) \\ Pontifícia Universidade Católica do Paraná, Curitiba-PR, Brasil \\ Pedro Guilherme Basso Machado (D) \\ Unibrasil, Curitiba-PR, Brasil \\ Juan José Camou Viacava (D) \\ Pontifícia Universidade Católica do Paraná, Curitiba-PR, Brasil
}

\section{RESUMO}

O objetivo do presente artigo é avaliar a estabilidade temporal do UWES, instrumento de avaliação do engajamento no trabalho, construto caracterizado por elevado vigor, dedicação e absorção no trabalho. A amostra foi composta por 423 trabalhadores de teleatendimento e utilizou-se a metodologia de teste/reteste mediante intervalo de tempo de um mês, com emprego de análises descritivas e de confiabilidade. Como resultado, o instrumento manteve qualidade psicométrica adequada (alfa de Cronbach $>0,8$; CFI $>0,95$; RMSEA $<0,07$ ) além de bons resultados que atestam a sua invariância de medida entre as coletas longitudinais (configural, métrica e escalar), dados alinhados com o manual do instrumento. Com relação à estabilidade temporal, houve acréscimo dos valores do alfa de Cronbach e correlações significativas e positivas. Sugere-se que os resultados obtidos denotam adequada qualidade psicométrica com estabilidade temporal, contudo ressalta-se a necessidade de novos estudos, em especial pela aplicação ter ocorrido em apenas uma categoria profissional, empresa e região.

Palavras-chave: engajamento no trabalho; UWES; invariância temporal; estabilidade temporal; psicometria.

\section{ABSTRACT - Work Engagement: Psychometric and Temporal Invariance Characteristics of the Utrecht Work Engagement Scale}

The aim of this study was to evaluate the temporal stability of the UWES instrument for the evaluation of work engagement. This construct is characterized by high vigor, dedication and absorption in the work. The sample was composed by 423 telemarketing workers and the test/retest methodology with an interval of 1 month was used. Descriptive and reliability analyses were carried out. The instrument presented adequate psychometric qualities (Cronbach's Alpha $>0.8$; CFI $>0.95 ;$ RMSEA $<0.07$ ) and showed good results regarding the Invariance Measurement between longitudinal samples (configural, metric and scalar), in agreement with data from the instrument's manual. Regarding the temporal stability there was an increase in Cronbach's Alpha values and Pearson's correlations were significant and positive. The results showed adequate psychometric quality with temporal stability, however, the need of future studies is emphasized, especially considering that the application was made using only one professional category, from one organization and region.

Keywords: work engagement; UWES; temporal invariance; temporal stability; psychometry.

\section{RESUMEN - Compromiso Laboral: Características Psicométricas y Invarianza Temporal del UWES}

El objetivo del presente artículo es evaluar la estabilidad temporal del UWES, instrumento de evaluación del compromiso laboral, constructo caracterizado por elevado vigor, dedicación y concentración en el trabajo. La muestra fue compuesta por 423 trabajadores de teleoperación y se utilizó el método test/retest mediante un intervalo de tiempo de un mes, con empleo de análisis descriptivos y de confiabilidad. Como resultado, el instrumento mantuvo calidad psicométrica adecuada (alfa de Cronbach $>0,8$; CFI $>0,95$; RMSEA <0,07) además de buenos resultados que atestiguan su invarianza de medida entre las colectas longitudinales (configural, métrica y escalar), datos alineados con el manual del instrumento. Con respecto a la estabilidad temporal, hubo acrecimiento de los valores del alfa de Cronbach y correlaciones significativas y positivas. Se sugiere que los resultados obtenidos denotan una adecuada calidad psicométrica con estabilidad temporal, sin embargo, se resalta la necesidad de nuevos estudios, especialmente por la aplicación solo haber sido realizada en una categoría profesional, una empresa y región determinada.

Palabras clave: compromiso laboral; UWES; invarianza temporal; estabilidad temporal; psicometría.

Historicamente, a Psicologia priorizou o estudo dos transtornos, sintomas e doenças, ou seja, aspectos considerados negativos em detrimento dos aspectos positivos da saúde (Pocinho \& Perestrelo, 2011; Salanova \& Schaufeli, 2009; Schaufeli, 2015). Como resposta a essa tendência, surgiu o movimento denominado Psicologia 
Positiva, que busca privilegiar os aspectos salutares em detrimento dos enfermos. Nesse contexto, encontra-se o engajamento no trabalho como construto diretamente relacionado à saúde ocupacional (Salanova \& Schaufeli, 2009; Schaufeli \& Bakker, 2003).

Esse construto é compreendido como um estado cognitivo positivo, sempre relacionado ao trabalho, persistente no tempo, de natureza motivacional e social, não focado em um único objetivo ou situação (Harju, Hakanen, \& Schaufeli, 2016; Porto-Martins, Machado, \& BenevidesPereira, 2013; Schaufeli, 2012, 2015; Yulita, Dollard, \& Idris, 2017). O tema é considerado recente e com crescente interesse na literatura (Kulikowski, 2017; Schaufeli, 2012). Inclusive, no contexto brasileiro, pode ser considerado pouco explorado (Oliveira \& Rocha, 2017).

O engajamento no trabalho é tridimensional, caracterizado por um fator comportamental e energético, representado por elevados níveis de energia e resiliência (dimensão Vigor); um fator emocional de senso de significância e desafio (dimensão Dedicação); e um cognitivo de elevada concentração e absorção nas atividades (dimensão Absorção) (Schaufeli, 2012, 2016, 2017; Yulita et al., 2017). O engajamento é tanto individual como coletivo, uma vez que está intimamente conectado ao desempenho organizacional (Acosta, Salanova, \& Llorens, 2011; Salanova \& Schaufeli, 2009; Schaufeli, 2012).

Trata-se de um construto importante para o ambiente organizacional, uma vez que é preditivo de boa performance e satisfação profissional (Pocinho \& Perestrelo, 2011; Porto-Martins et al., 2013; Salanova \& Schaufeli, 2009). Em síntese, considera-se que o engajamento reforça os efeitos dos recursos organizacionais disponíveis em relação ao trabalho em direção ao incremento no desempenho organizacional (Porto-Martins et al., 2013; Salanova \& Schaufeli, 2009; Schaufeli, 2015).

Profissionais com elevado engajamento (engaged) em suas atividades profissionais geralmente possuem melhor saúde física e psicológica, trabalham com afinco (Vigor), são imersos em suas atividades (Dedicação) e sentem-se altamente concentrados em seus trabalhos (Absorção) (Bakker \& Leiter, 2010; Porto-Martins et al., 2013), assim como apresentam menor rotatividade (Oliveira \& Rocha, 2017). Ou seja, potencializam-se os aspectos positivos tanto do trabalhador como da organização.

Uma vez que o engajamento no trabalho pode ser considerado como um fator importante para as organizações, faz-se necessária a utilização de instrumentos com boa qualidade psicométrica para avaliar o construto e realizar pesquisas referentes à saúde laboral. Ainda, o incremento da validação desse construto fornecerá uma importante ferramenta para avaliações e estratégias organizacionais (Salanova \& Schaufeli, 2009; Schaufeli, 2016).

Cabe destacar que há distintas formas de avaliar o engajamento no trabalho, por exemplo, os autores Maslach e Leiter (2008) apontam que o engajamento pode ser aferido como oposto direto das três dimensões do Burnout, que se caracterizam por energia, comprometimento e eficácia profissional. Nessa perspectiva, o construto é avaliado pelo padrão oposto das pontuações das três dimensões do Burnout aferido pelo Maslach Burnout Inventory - MBI (Maslach, Jackson, \& Leiter, 1996). Contudo, os autores Salanova, Schaufeli, Llorens, Peiró e Grau (2000); Salanova e Schaufeli (2009) defendem que utilizar o MBI para avaliar o engajamento no trabalho é um procedimento inadequado, devendo-se utilizar instrumento específico para esse fim.

Nesse sentido, foi escolhido o instrumento do Utrecht Work Engagement Scale (UWES) versão de 17 itens para trabalhadores, de Schaufeli e Bakker (2003), uma vez que esse foi o primeiro instrumento criado para avaliar especificamente o engajamento no trabalho (Salanova et al., 2000), possui versões em 19 idiomas contando com uma base de dados de mais de 30.000 sujeitos (Kulikowski, 2017; Salanova \& Schaufeli, 2009; Schaufeli \& Bakker, 2010) e, principalmente, por ser considerado como instrumento de referência para essa aferição (Salanova \& Schaufeli, 2009). O UWES pode ser utilizado tanto na forma unifatorial como trifatorial, nesta última se considera os três aspectos do engajamento: vigor, dedicação e absorção (Salanova \& Schaufeli, 2009). Ainda, destaca-se que o modelo trifatorial apresenta maior adequação nos índices de ajuste (Salanova et al., 2000; Schaufeli, Martínez, Marques-Pinto, Salanova, \& Bakker, 2002; Schaufeli \& Bakker, 2003; Schaufeli \& Bakker, 2010).

O objetivo do presente artigo é avaliar a estabilidade temporal do UWES, uma vez que não se encontram publicações com esse tipo de análise no contexto brasileiro. Para tanto, foram realizados testes de validade (fatoriais confirmatórias nos modelos uni e trifatorial), invariância Configural, invariância Métrica e de Invariância Escalar) para atestar sua estabilidade temporal. Isso foi feito para em seguida poder verificar (Damásio, 2013) a oscilação dos escores (médias), intensidade da força entre as dimensões (correlações de Pearson e Intraclasse), confiabilidade (alfas de Cronbach), (teste e reteste, diferenças de média $t$ de Student. Ou seja, pretende-se contribuir com o uso e disseminação do construto e de seu respectivo instrumento.

Com relação a estabilidade temporal destaca-se que essa técnica de aferição psicométrica é um fator relevante, uma vez que esse tipo de análise é considerado pertinente quando se pretende discutir a qualidade de um instrumento, sendo uma forma de avaliação de confiabilidade e de consistência de medidas de escalas. Ou seja, implica em uma forma de aprimorar análises psicométricas, evitar erros e avaliar temporalmente dados de uma determinada escala (Hair, Black, Babin, \& Anderson, 2010).

Atualmente, o instrumento conta com três versões traduzidas para amostra brasileira, conforme descrito: 
1. Versão realizada por Porto-Martins e BenevidesPereira (2008), que é apresentada no manual da versão brasileira do instrumento (Agnst, Benevides-Pereira, \& Porto-Martins, 2009) e que segue a mesma estrutura da versão original em inglês de Schaufeli e Bakker (2003). Essa versão, com 17 itens, possui publicações, como as de Machado (2010) e Machado, Porto-Martins e Benevides-Pereira (2014), trabalhos complementares com amostra de $N=212$ trabalhadores do transporte coletivo; Machado, Porto-Martins e Amorim (2012) com $N=63$ professores; Machado e Porto-Martins (2013) e Machado e Porto-Martins (2015), ambos com amostra multiocupacional de $N=701$ profissionais do setor industrial; Freitas e Charão-Brito (2017), com $N=40$ profissionais multidisciplinares de saúde; Lourenção (2018) com 15 gestores de Unidades de Saúde da Família; De Mattos, Andrade e Tayse (2019) com 92 bancários; Teixeira et al. (2017), com N=36 médicos e Gonsalez et al. (2017), com $N=82$ profissionais de programas de aprimoramento e aperfeiçoamento profissional em saúde.

2. Versão de Vazquez, Magnan, Pacio, Hutz e Schaufeli (2015), que também segue a versão original do UWES com 17 itens e apresenta estudos, como o próprio Vazquez et al. (2015) e Magnan, Vazquez, Pacico e Hutz (2016), ambos com $N=1.167$ trabalhadores de cinco regiões do Brasil; Dalanhol, Freitas, Machado, Hutz e Vazquez (2017), com N=200 Oficiais de Justiça Federal, de Porto Alegre e região metropolitana; Ferreira et al. (2016), com $N=2.897$ sujeitos provenientes de cinco pesquisas diferentes; Mercali e Costa (2019) com 506 professores de todas as unidades federativas do Brasil e Oliveira e Rocha (2017), em estudo com $N=299$ profissionais do setor de economia, estes dois últimos utilizaram a versão reduzida de nove itens.

3. Versão dos autores Siqueira, Martins, Lima, Agapito e Souza (2011), instrumento bidimensional com as dimensões Vigor e Dedicação, ambas com cinco itens e escala tipo Likert de 1 a 5 . Instrumento que conta com investigações, como as de Caldas, Somensari, Costa, Siqueira e Claro (2013), com amostra de $N=71$ docentes; Cavalcanti (2013) e Cavalcanti, Siqueira e Kuniyoshi (2015), ambos com $N=159$ profissionais da área de gestão de pessoas e Martinsa, Costa e Siqueira (2015), com N=175 trabalhadores da região metropolitana de São Paulo.

Destaca-se que a versão escolhida para o presente estudo foi a de Porto-Martins e Benevides-Pereira (2008), que está presente na tradução do manual do UWES para o português (Agnst et al., 2009). Versões do manual e do instrumento que se encontram disponível no site oficial do autor do instrumento (http://www.wilmarschaufeli.nl/).

\section{Método}

\section{Participantes}

O estudo foi realizado com operadores de teleatendimento, uma vez que as empresas de telemarketing são um dos maiores fornecedores de emprego do país. Além da importância econômica, essa população se caracteriza comumente por apresentar alterações psicológicas mesmo em um curto período de trabalho (Rêgo, 2008).

A amostra foi constituída por $N=423$ operadores de teleatendimento de um total 500 profissionais $(84,6 \%$ de taxa de resposta) de uma empresa específica da área de telecomunicação situada no sul do Brasil. Com relação aos dados obtidos, a maioria eram mulheres $81,8 \%(n=346)$, com média de idade de 22 anos, com até um ano de atuação na empresa 83,7\%; $(n=354)$, escolaridade de nível médio completo 86,8\% (367), relacionamento afetivo estável 52,7\% $(n=223)$ e sem filhos, 71,4\% $(n=302)$.

\section{Instrumentos}

Referente aos instrumentos, utilizou-se questionário sociolaboral elaborado pelos autores com a finalidade de averiguar características dos trabalhadores e o Utrecht Work Engagement Scale (UWES) de Wilmar Schaufeli e Arnold Bakker (2003) versão brasileira de Porto-Martins e Benevides-Pereira (2008).

Esse instrumento é formado por 17 itens e utiliza escala tipo Likert de sete pontos. Os resultados são categorizados em cinco níveis que variam desde "muito baixo" até "muito alto". O UWES avalia tanto o índice de Engajamento Geral, assim como três escalas específicas constitutivas do construto, especificadas a seguir, de acordo com Salanova e Schaufeli (2009) e Schaufeli e Bakker (2003):

Vigor (VI): seis itens, como "Em meu trabalho, sinto-me repleto - cheio - de energia".

Dedicação (DE): cinco itens, como "Eu acho que o trabalho que realizo é cheio de significado e propósito".

Absorção ( $\mathrm{AB})$ : seis itens, como "O tempo voa quando estou trabalhando".

\section{Procedimentos}

A aplicação dos instrumentos foi previamente autorizada pelos gestores e realizada por meio de questionário eletrônico pela ferramenta Qualtrics. Como todos os operadores possuíam computador em suas baias (local de trabalho), foi gerado um link e inserido ao desktop de todos os colaboradores. $\mathrm{Na}$ data da primeira aplicação, os pesquisadores apresentaram a intenção da pesquisa, passaram o Termo Livre e Esclarecido e, aqueles que aceitaram participar, acessaram o link indicado.

O controle da amostra para o teste/reteste foi feito por meio de uma identificação numérica, valor gerado automaticamente via login dos participantes, respeitando-se assim o sigilo e controle deles. Destaca-se que as aplicações ocorreram todas ao mesmo instante, ou seja, todos os participantes preencheram o formulário ao mesmo tempo em seus respectivos desktops. Deu-se um intervalo de 1 mês e o procedimento foi repetido. 
Ressalta-se ainda que o presente manuscrito faz parte de uma tese doutoral que teve o respectivo projeto devidamente submetido e aprovado em um comitê de ética de uma instituição de nível superior.

\section{Análise de Dados}

Para análises das provas estatísticas, utilizaram-se os programas SPSS e AMOS, ambos na versão 23, para realizar o cálculos supracitados no objetivo deste manuscrito considerando como parâmetros: alfa de Cronbach >0,70 (Hair et al., 2010); Coeficiente de Correlação Intraclasse (CCI) próximo a 01 (Nakagawa, Johnson, \& Schielzeth, 2017); Qui-quadrado/graus de liberdade (CMIN/DF) <5,0 (Pimentel, Gouveia, \& Fonseca, 2005); Erro Quadrático Médio de Aproximação (RMSEA) <0,80 (Byrne, 2010; Hooper et al., 2008); Índice que verifica a Proximidade do Ajuste (PCLOSE <0,05 (Hair et al, 2010); Índice de Bondade Ajuste do Modelo (AGFI $>0,80$ (Hair et al, 2010); Índice de Ajuste Comparativo (CFI) $>0,90$ (Hair et al, 2010) e Índice de Regressão Padronizada (beta) $>0,40$ (Hair et al, 2010). Índice comparativos de modelos (ECVI quanto menos, maior fit (Brown, 2006)

Para averiguar a confiabilidade por meio da estabilidade temporal, utilizou-se o intervalo de tempo de 30 dias, conforme defendido pelos autores Renosto, Biz, Hennington e Pattussi (2009) que destacam que esse intervalo atende aos critérios de que diferentes aplicações não devem ser demasiadamente próximas, pois podem afetar os dados pelo efeito memória, mas também não podem ser excessivamente longas, pois comprometem a interpretação dos resultados. Ainda, esse intervalo de tempo está em sintonia com o defendido por Malhotra (2012) que esclarece que o período de tempo deve oscilar entre duas a quatro semanas. Sendo assim, utilizou-se o intervalo no limite do tempo sugerido por Malhotra (2012) para aumentar a segurança das análises uma vez que quanto maior o período, menor a probabilidade de confiança.

Com o intuito de facilitar as análises, realizou-se a seguinte categorização: Grupo Total $(N=423)$, composto por todos os sujeitos que responderam ao questionário, para aqueles que preencheram ambas as aplicações (teste/ reteste) considerou-se somente a primeira aplicação. A segunda categorização denominou-se de Grupo Teste/ Reteste $(n=275)$, que representa todos os operadores de teleatendimento que preencheram ambas as aplicações, tanto no teste como no reteste.

\section{Resultados}

No que tange às análises fatoriais confirmatórias (AFC), foram avaliados os modelos uni e trifatorial. Os resultados do modelo trifatorial, não recursivo de primeira ordem (conforme figura 1), utilizando como amostra o grupo total foram $\mathrm{CMIN} / \mathrm{DF}=3,00$; RMSEA $=0,07 ; \quad$ SRMR $=0,03 ; \quad$ PCLOSE $=0,00$; AGFI $=0,89, \mathrm{CFI}=0,96$ e ECVI=1,150. Já os resultados do modelo unifatorial foram $\mathrm{CMIN} / \mathrm{DF}=3,50$; RMSEA $=0,08 ; \quad$ SRMR $=0,04 ; \quad$ PCLOSE $=0,00$; $\mathrm{AGFI}=0,86, \mathrm{CFI}=0,94$ e $\mathrm{ECVI}=1,485$.

De acordo com a Figura 1, nota-se que a correlação estimada obteve o valor máximo de 1,00 entre as dimensões $\mathrm{AB} / \mathrm{UWES} \leftrightarrow \mathrm{VI} / \mathrm{UWES}$, assim como se observa o valor de 0,98 entre $\mathrm{AB} / \mathrm{UWES} \leftrightarrow$ DE/UWES e 0,98 entre DE/UWES $\leftrightarrow$ VI/UWES. Complementando esses dados, as covariâncias estimadas foram de: $\mathrm{AB} / \mathrm{UWES}$ $\leftrightarrow$ VI/UWES: 1,93, com erro padronizado de 0,17; $\mathrm{AB} /$ UWES $\leftrightarrow$ DE/UWES: 1,91, com erro padronizado de 0,$17 ; \mathrm{DE} / \mathrm{UWES} \leftrightarrow \mathrm{VI} / \mathrm{UWES} 1,94$, com erro padronizado de 0,17 .

Os dados supracitados evidenciam a forte relação entre as dimensões, o que está coerente com o marco teórico que possibilita a utilização unifatorial do UWES. Esses resultados foram confirmados quando verificada a Correlação interna de Pearson, que foi positiva, elevada e significativa $(p<0,01)$, sendo de $r=0,89$ entre Dedicação e Vigor, $r=0,87$ para Absorção e Vigor e $r=0,85$ entre Absorção e Dedicação.

Com relação aos índices beta (Índice de Regressão Padronizada), verifica-se, na Figura 1, que o maior valor foi de $b=0,88$ simultaneamente entre os itens 04,05 e 10 e suas respectivas escalas. Por outro lado, os menos intensos foram entre os itens $16(b=0,36)$ e $13(b=0,57)$ ficando somente o item 16 abaixo do critério ideal, dado corroborado pelo manual da UWES (Schaufeli e Bakker, 2003) e também por Ramalho, Teles, Ramalho e Ribeiro (2017). De forma geral, esses dados sugerem a elevada relação dos itens frente às suas respectivas dimensões.

Ainda, para atestar a estabilidade temporal da escala, foram realizados testes de invariância de medida considerando as possíveis diferenças entre os dados longitudinais (amostra teste e reteste). Conforme recomendação de Milfont e Fischer (2010), Damásio (2013), Putnick e Bornstein (2016) executaram-se os testes de Invariância Configural, Invariância Métrica e de Invariância Escalar. Para executar essas análises, o primeiro passo é determinar o modelo de análise longitudinal. Alguns autores, como Kline (2011) e Chungkham, Ingre, Karasek, Westerlund, \& Theorell (2013) apontam que, para apenas duas coletas podem ser realizados testes de comparação entre grupos (grupo $1=$ teste, grupo $2=$ reteste) devido sua facilidade de análise. Entretanto esse tipo de análise tem o viés de desconsiderar a relação entre as duas observações intraindividual, requisito necessário não só para atestar a invariância do modelo, mas também necessário para realizar testes de comparações diretas de médias (como testes $t$ pareados e anovas repetidas) (Little, Bovaird, \& Slegers, 2006; Duncan \& Duncan, 2009). 


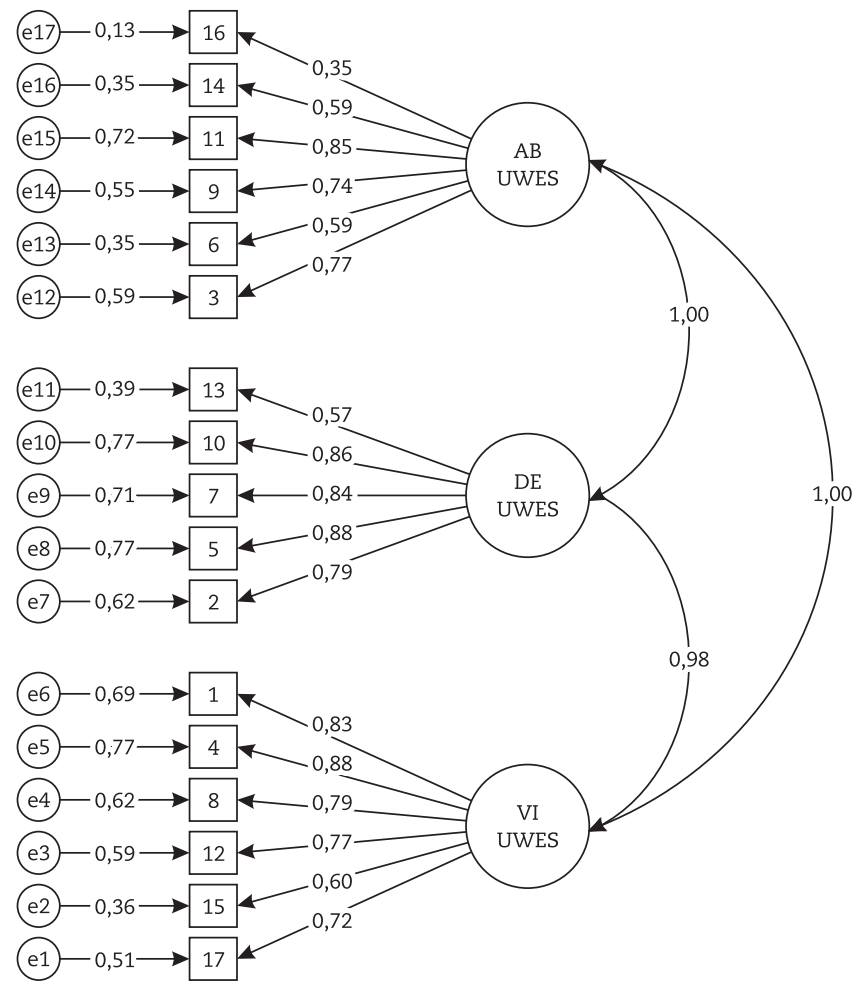

Figura 1. Modelo não Recursivo de Primeira Ordem do UWES

Segundo Duncan e Duncan (2009) um dos modelos usualmente recomendados seria o modelo LGM (Latent Growth Modeling - ou Latent Variable Growth Modelling), pois seria capaz de analisar as variações de múltiplas observações sobre os mesmos sujeitos ao longo do tempo e, assim, determinar variações não só das médias, mas também dos interceptos e coeficientes angulares. Entretanto, os autores (Duncan \& Duncan, 2009; Kievit et al., 2018) apontam que esse tipo de modelo não seria ideal para apenas duas mensurações pela incapacidade de medir a forma da variação e, assim, poderia produzir vieses de análise. De acordo com Little et al. (2006) e Little (2013), é possível verificar variações de mudanças intra e interindividuais a partir de técnicas derivadas de métodos de variáveis latentes. De acordo com esses autores e Kievit et al. (2018), o método recomendado nesses casos seria o LCS (Latente Change Score Models) algumas vezes também chamado de LDS (Latent Diference Score Models). Isso seria recomendado, pois as diversas limitações de outras abordagens (como falta de confiabilidade e problemas de erros correlacionados) seriam remediadas pelos seus procedimentos, além de apresentar maior versatilidade e flexibilidade (Kievit et al., 2018).

De forma geral, os modelos LCS podem considerar variações duas ou mais observações diretas e, variações de variáveis latentes com múltiplos indicadores em cada coleta (aqui, teste e reteste). Com esse processo seria produzido uma variância em cada dimensão em que o erro seria livre e, como resultado, a variância entre observações (delta) seria mensurada sem erros. Nesses modelos especificados, a variância dos fatores exógenos na primeira coleta (tempo 1, ou "teste") e as variações (especificadas por uma variável latente delta) são fixados em 1 (um) e, uma mensuração de correlação de erro seria verificada entre essas duas de forma livre. Ainda, a variável latente de resultado da segunda coleta (tempo 2, ou "reteste") seria resultante - além de seus indicadores observados - também da variável latente Tempo 1 e da variação delta com coeficientes de regressão fixados em 1 (um). Adicionalmente, devem ser permitidas a covariância residual dos erros dos termos de cada observação e dimensão e, deve ser adicionados "erros correlacionados" (Kievit et al., 2008)

A partir desses procedimentos, duas abordagens são possíveis para o construto aqui proposto que possui três dimensões para o engajamento. Uma abordagem seria a de variação de múltiplas variáveis latentes em que cada dimensão da escala (ABUWES, DEUWES e VIUEWS) da primeira coleta devem ser comparadas com a variação Delta (DeltaABUWES, DeltaDEUWES, DeltaVIUEWS) com suas respectivas novas mensurações no tempo, da segunda coleta. A segunda abordagem é a de variação de múltiplos indicadores que formam uma única variável latente em que o construto latente Engajamento seria comparado com suas variações antes e depois (teste e 
reteste). Apesar de resultados semelhantes de índices de ajustes (índices do modelo longitudinal de variação deste única varável latente Engajamento: $\mathrm{CMIN} / \mathrm{DF}=2,067$; SRMR $=0,08 ; \quad$ RMSEA $=0,062 ; \quad$ PCLOSE $<0,001$, $\mathrm{AGFI}=0,80, \mathrm{CFI}=0,927$ e $\mathrm{ECVI}=4,467)$ como consideramos o modelo com trifatorial e, assim, devido a necessidade de comparações das médias latentes entre as três dimensões do construto, a primeira abordagem foi a escolhida.

O primeiro procedimento para verificar a invariância de medida seria o de invariância Configural, testado por um modelo irrestrito (unconstrained model) em que a estrutura de um determinado instrumento é plausível para determinados grupos ou condições configuradas a priori (Cheung \& Rensvold, 2002; Damásio, 2013). Após configurar o modelo conforme recomendações de Little et al. (2006), Little (2013) e Kievit et al. (2018), seus índices foram considerados satisfatórios (CMIN/DF $=2,109$; SRMR $=0,08 ; \quad$ RMSEA $=0,064 ; \quad$ PCLOSE $<0,001$, $\mathrm{AGFI}=0,817, \mathrm{CFI}=0,924$ e $\mathrm{ECVI}=4,544)$ indicando a invariância Configural.
No segundo, teste de Invariância Métrica (também denominado teste de cargas fatoriais), é investigado se os pesos de regressão dos itens (cargas fatoriais) seriam equivalentes para as condições avaliadas, comparando o modelo Irrestritro (modelo Configural longitudinal) com um modelo totalmente Irrestrito (Cheung \& Rensvold, 2002; Damásio, 2013; Putnick \& Bornstein, 2016). Para esse modelo totalmente restrito (fully constrained) devem ser retiradas as restrições de Pesos das Regressões e, posteriormente, inserindo-as nas Variáveis Latentes e nomeando os parâmetros de pesos das regressões igualmente entre as condições. Logo após, seguindo a recomendação de Verdan \& Oort (2014), deve ser analisado se existe uma deterioração dos indicadores. O modelo com os ajustes para verificar a Invariância Métrica apresentou indicadores satisfatórios $(\mathrm{CMIN} / \mathrm{DF}=1,888$; $\mathrm{SRMR}=0,07$; RMSEA $=0,057 ; \quad$ PCLOSE $=0,021, \quad$ AGFI $=0,833$ $\mathrm{CFI}=0,940$ e ECVI=4,138). A análise comparativa desses indicadores não apontou uma deterioração, mas sim, uma pequena melhora (Tabela 1), indicando sua invariância Métrica (Little, 2013; Verdan \& Oort, 2014).

Tabela 1

Comparação das Cargas Fatoriais entre os Modelos

\begin{tabular}{cccc}
\hline Dimensão & Item & Teste & Reteste \\
\hline & UWES16 & 0,508 & 0,619 \\
& UWES14 & 0,593 & 0,867 \\
ABUWES & UWES11 & 0,803 & 0,738 \\
& UWES9 & 0,706 & 0,733 \\
& UWES6 & 0,588 & 0,801 \\
& UWES3 & 0,715 & 0,723 \\
& UWES13 & 0,860 \\
& UWES10 & 0,547 & 0,906 \\
& UWES7 & 0,847 & 0,910 \\
& UWES5 & 0,828 & 0,828 \\
& UWES2 & 0,848 & 0,883 \\
& UWES1 & 0,741 & 0,906 \\
& UWES4 & 0,711 & 0,818 \\
& UWES8 & 0,844 & 0,807 \\
& UWES12 & 0,739 & 0,697
\end{tabular}

O último teste, de Invariância Escalar, é necessário para assegurar que os escores obtidos sejam "totalmente relacionados ao nível de traço latente dos sujeitos, independente de seu grupo ou observação" (Damásio, 2013, p. 214). Isso deve ser feito ao restringir o intercepto para ser equivalente nas duas condições e, também, com as restrições no modelo de invariância métrica (Putnick \& Bornstein, 2016). Nesse último, foi verificado uma pequena deterioração do CFI e do ECVI $(\mathrm{CMIN} / \mathrm{DF}=2,288 ; \quad \mathrm{SRMR}=0,08 ; \quad \mathrm{RMSEA}=0,069$; PCLOSE $<0,001$, CFI $=0,908$ e ECVI=5,147 - AGFI não gerado nesse modelo) mais ainda dentro do satisfatório, indicando sua invariância escalar assim como nos demais testes (Tabela 2). 
Tabela 2

Comparação dos Índices de Ajuste entre os Modelos

\begin{tabular}{|c|c|c|c|c|c|c|c|}
\hline & CMIN/DF & SRMR & RMSEA & PCLOSE & AGFI & CFI & ECVI \\
\hline $\begin{array}{l}\text { Trifatorial } \\
\text { (amostra total } n=423 \text { ) }\end{array}$ & 3,000 & 0,03 & 0,07 & 0,001 & 0,89 & 0,96 & 1,150 \\
\hline $\begin{array}{l}\text { Unifatorial } \\
\text { (amostra total } n=423 \text { ) }\end{array}$ & 3,503 & 0,04 & 0,08 & 0,001 & 0,86 & 0,94 & 1,485 \\
\hline $\begin{array}{l}\text { Longitudinal }(n=275) \\
\text { (Inv. Configural) }\end{array}$ & 2,109 & 0,08 & 0,06 & 0,001 & 0,82 & 0,93 & 4,544 \\
\hline $\begin{array}{l}\text { Longitudinal ( } n=275) \\
\text { Regressões Restritas (Inv. Métrica) }\end{array}$ & 1,888 & 0,07 & 0,06 & 0,021 & 0,83 & 0,94 & 4,138 \\
\hline $\begin{array}{l}\text { Longitudinal }(n=275) \\
\text { Intercepto e Regressões Restritas }\end{array}$ & 2,288 & 0,08 & 0,07 & 0,001 & - & 0,91 & 5,147 \\
\hline (Inv. Escalar) & & & & & & & \\
\hline
\end{tabular}

Devido a essa pequena deterioração também foi feita a análise de comparação considerando as amostras teste e reteste como grupos, seguindo a recomendação de Kline (2011) e Chungkham et al. (2013). Como é feita uma comparação entre grupos, o principal indicador é se não houve uma diferença significativa entre os índices de qui-quadrado e graus de liberdade entre as condições. Mais uma vez foi verificada a invariância Configural devido seus bons índices (CMIN/DF $=2,81 ; \mathrm{SRMR}=0,04$; RMSEA $=0,06 ; \quad$ PCLOSE $=0,009 ; \quad$ AGFI $=0,83$, $\mathrm{CFI}=0,94$; $\left.\mathrm{ECVI}=1,460 / \chi^{2}(232)=652,1\right)$, assim como a invariância Métrica comparativa com o modelo fully constrained $\left[\chi^{2}(249)=665,1\right.$, comparação: $\left.p=0,74\right]$ e também a invariância Escalar $\left[\chi^{2}(278)=692,7\right.$ / comparação: $p=0,70]$. Dessa forma, independentemente do método, foi verificada a invariância de medida do construto Engajamento e suas três dimensões.

Uma primeira comparação da variação temporal das médias das dimensões, utilizando o modelo longitudinal do construto latente Engajamento, apresentou variação significativa da média geral (UWES Média Estimada $=3,593$; $\mathrm{SE}=0,090$; deltaUWES: média estimada $=-0,248 ; \mathrm{SE}=0,103 ; p=0,016)$ indicando uma tendência de queda das médias de Engajamento. Ao analisar a variação de cada uma das dimensões, utilizando o modelo longitudinal (do modelo de invariância Configural com as três dimensões), foi verificado que as duas dimensões DEUWES e VIUWES tiveram variação negativa significativa entre as coletas (DEUWES: Média estimada=2,888; SE: 0,092; deltaDEUWES: média estimada $=-0,219 ; \mathrm{SE}=101 ; p=0,017 / \mathrm{VIUWES}$ : Média estimada $=3,159$; $\mathrm{SE}=0,80$; deltaVIUWES: média estima$\mathrm{da}=-0,418 ; \mathrm{SE}=0,084 ; p<0,001)$, enquanto a dimensão ABDUWES teve variação apenas próxima de marginal (ABDUWES: Média estimada=2,735 SE:0,094/delta$\mathrm{AB}=-0,153 ; \mathrm{SE}=0,101 ; p=0,130)$. Depois disso, seguiu-se para a análise descritiva e comparação das médias entre as coletas e comparativa com outros estudos.

Referente às essas análises realizadas, quando comparadas as médias ponderadas do UWES do presente estudo, com os dados da amostra multiocupacional e internacional $(N=12.161)$ fornecidos pelo Manual do Instrumento (Schaufeli \& Bakker, 2003), constata-se que o Grupo Total obteve valores inferiores a esse parâmetro para todas as dimensões: VI $(M=3,46 ; D P=1,39$ versus $M=4,24 ; D P=1,09$ para o Manual), $\mathrm{DE}(M=3,49$; $D P=1,49$ versus $M=4,33 ; D P=1,36$ para o Manual), $\mathrm{AB}(M=3,05 ; D P=1,29$ versus $M=3,77 ; D P=1,28$ para o Manual) e UWES Geral $(M=3,32 ; D P=1,32$ versus $M=4,10 ; D P=1,11$ para o Manual), conforme valores apresentados na Tabela 3. Cabe destacar que não há estudos realizados com operadores de teleatendimento em relação ao engajamento no trabalho no Brasil.

Em relação às médias das distintas aplicações, os dados ilustrados na Tabela 3 apontam o decréscimo dos índices das três dimensões e da escala Geral do Teste para o Reteste. Esses dados confirmam o preconizado em diversos estudos que encontraram que a atividade de teleatendimento é considerada como desgastante, rigidamente controlada e que o tempo laborado na atividade é uma variável importante, que incrementa o sofrimento desses trabalhadores (Leon \& Halbesleben, 2013; Rechenberg, Goulart, \& Roithmann, 2011; Rêgo, 2008).

Referente ao impacto das diferenças de médias em relação ao intervalo de tempo de um mês, testes $t$ pareados indicaram que houve uma variação da média geral (Mantes=3,22; DP=1,27/Mdepois=2,78; $p<0,001)$ e também alterações significativas para as três dimensões $(p<0,01)$, com decréscimo do engajamento na segunda aplicação. Seguindo o padrão das variações das médias latentes, a oscilação mais intensa foi para o Vigor $(t=6,25 /$ $p<0,01)$, seguida de Dedicação $(t=6,07 / p<0,01)$. Ainda que nos testes de médias de varáveis latentes o resultado tenha sido próximo de marginal, o teste $t$ pareado 
apontou para uma variação significativa da Absorção $(t=5,62 / p<0,01)$, a menor das três dimensões. Dessa forma, é reforçada a premissa teórica de que essa atividade é desgastante para o trabalhador.

Com relação à confiabilidade, o UWES apresentou índices de alfa de Cronbach satisfatórios para as três dimensões, assim como para a escala Geral, uma vez que foram superiores a 0,70 , conforme Tabela 3. Dados em concordância com outros estudos que utilizaram essa versão do UWES, como os de De Mattos et al. (2019); Gonsalez et al. (2017); Lourenção (2018); Machado (2010); Machado e Porto-Martins (2013); Machado et al. (2014); Machado et al. (2012); Machado e Porto-Martins (2013); Machado e Porto-Martins (2015) e Teixeira et al. (2017).

Ainda na Tabela 3, é possível verificar que o alfa de Cronbach apresentou elevação da primeira aplicação para a segunda, nas três dimensões, o que também é considerado bom indicativo psicométrico. Esses resultados estão em sintonia com outras pesquisas com intervalo de tempo similar, como as de Renosto et al. (2009) e Silva e Coutinho (2005), que defendem essa oscilação como indício de qualidade adequada do instrumento.

Referente à correlação de Pearson entre as dimensões no teste/reteste, os dados foram todos positivos e significativos $(p<0,01)$ e variaram desde $r=0,57$ (Dedicação Teste/Dedicação Reteste) até $r=0,59$ (Absorção Teste/ Absorção Reteste). Esses índices evidenciam forte relação entre as aplicações, o que é considerado como bom indicativo de confiabilidade e estabilidade temporal (Hjermstad, Fossa, Bjordal, \& Kaasa, 1995). Para complementar essa discussão, foi verificado o Coeficiente de Correlação Intraclasse (CCI) entre as aplicações, estes resultados são apresentados na Tabela 4.

Tabela 3

Número de Itens, Média, Desvio Padrão e Alfas dos Grupos Total, Teste e Reteste

\begin{tabular}{|c|c|c|c|c|c|c|c|c|c|c|}
\hline \multirow{2}{*}{ Dimensão } & \multirow{2}{*}{ Itens } & \multicolumn{3}{|c|}{$\begin{array}{c}\text { Grupo Total } \\
N=423\end{array}$} & \multicolumn{3}{|c|}{$\begin{array}{c}\text { Teste } \\
N=276\end{array}$} & \multicolumn{3}{|c|}{$\begin{array}{l}\text { Reteste } \\
N=276\end{array}$} \\
\hline & & Média & $\mathrm{DP}$ & Alpha & Média & $\mathrm{DP}$ & Alpha & Média & $\mathrm{DP}$ & Alpha \\
\hline VI/UWES & 6 & 3,46 & 1,39 & 0,89 & 3,37 & 1,34 & 0,88 & 2,90 & 1,37 & 0,90 \\
\hline DE/UWES & 5 & 3,49 & 1,49 & 0,89 & 3,39 & 1,42 & 0,87 & 2,89 & 1,45 & 0,90 \\
\hline AB/UWES & 6 & 3,05 & 1,29 & 0,82 & 2,97 & 1,26 & 0,81 & 2,59 & 1,24 & 0,83 \\
\hline GERAL/UWES & 17 & 3,32 & 1,32 & 0,95 & 3,23 & 1,27 & 0,95 & 2,79 & 1,28 & 0,95 \\
\hline
\end{tabular}

Tabela 4

Coeficiente de Correlação Intraclasse das Dimensões para Teste/Reteste

\begin{tabular}{|c|c|c|c|c|c|c|}
\hline & & \multirow{2}{*}{ Correlação Intraclasse } & \multicolumn{2}{|c|}{ IC 95\% } & \multicolumn{2}{|c|}{ Prova F } \\
\hline & & & L. inferior & L. superior & Valor & Sig. \\
\hline \multirow{2}{*}{ VI } & Medidas ${ }^{\text {ind. }}$ & $0,536 a$ & 0,405 & 0,639 & 3,631 & 0,000 \\
\hline & Medidas ${ }^{\text {pro }}$ & $0,698 c$ & 0,576 & 0,779 & 3,631 & 0,000 \\
\hline \multirow{2}{*}{$\mathrm{DE}$} & Medidas ${ }^{\text {ind. }}$ & $0,516 a$ & 0,387 & 0,619 & 3,410 & 0,000 \\
\hline & Medidas ${ }^{\text {pro }}$ & $0,681 c$ & 0,558 & 0,764 & 3,410 & 0,000 \\
\hline \multirow{2}{*}{$\mathrm{AB}$} & Medidas $^{\text {ind. }}$ & $0,560 a$ & 0,443 & 0,652 & 3,824 & 0,000 \\
\hline & Medidas ${ }^{\text {pro }}$ & $0,718 c$ & 0,614 & 0,790 & 3,824 & 0,000 \\
\hline
\end{tabular}

Em concordância com as correlações de Pearson, todos os CCIs apresentaram $p<0,01$. Esse dado reforça a perspectiva de que há relação significativa e positiva entre as duas aplicações do instrumento e fortalece o indicativo de estabilidade temporal adequada para essa versão do UWES.

O modelo do UWES foi considerado aceitável para a presente amostra, considerando o conjunto de análises realizadas. Ainda, os resultados estão em harmonia com os índices de ajuste apresentados no manual, tanto na amostra holandesa, $N=2.313$ como na internacional,
$N=12.161$ (Schaufeli \& Bakker, 2003), assim como para outros estudos internacionais (Schaufeli et al., 2002; Schaufeli \& Bakker, 2010).

Por fim, quando analisado o modelo unifatorial, este também apresentou índices adequados em relação aos parâmetros supracitados assim como convergentes com o manual do UWES. Ainda, quando comparado com o estudo de Machado e Porto-Martins (2013) que usou a mesma versão e modelo, obteve-se índices adequados e semelhantes $(\mathrm{CFI}=0,92$ versus 0,96 para o presente estudo; RMSEA $=0,10$ versus 0,07 para o presente estudo). 


\section{Discussões}

$\mathrm{O}$ aspecto central deste manuscrito foi a verificação da estabilidade temporal do UWES, em especial pelo fato de não ter sido encontrada nenhuma publicação com essa aferição do instrumento. Para tanto, utilizou-se o intervalo de um mês e foram realizados diversos testes estatísticos entre as aplicações, como: Correlação de Pearson, Coeficiente de Correlação Intraclasse, alfa de Cronbach, teste de Invariância Configural, Invariância Métrica e de Invariância Escalar. Ainda que não se tenha estudos prévios de estabilidade temporal do UWES, esperava-se que todas as análises fossem satisfatórias, uma vez que o instrumento é considerado adequado em diversos contextos, idiomas e regiões. Pressuposto confirmado, uma vez que o instrumento obteve índices satisfatórios para todas as verificações realizadas, fato que evidencia a confiabilidade da escala e apresenta consistência de resposta entre dois pontos no tempo, denotando que a medida de engajamento do UWES é confiável dentro dessa oscilação temporal.

Quando analisados os modelos tri e unifatorial, os resultados também denotaram índices adequados para ambos os casos, com o modelo trifatorial apresentando resultados levemente mais ajustados (índices CMIN/ DF RMSEA, AGFI e CFI). Dados que corroboram a possibilidade de uso unifatorial (escala Geral) assim como das três dimensões. Um fator resultante nessas análises que merece ser comentado é a alta correlação entre as dimensões do construto. Da mesma forma como apontando no manual UWES, foi verificado que a correlação entre as três dimensões é forte. Isso ocorreria em si porque, assim como as variáveis latentes representam os "verdadeiros escores" que estariam livres da medição de erro, a correlação entre os escores latentes devem ser, por definição, maiores que as correlações entre os escores observados. Também congruente com o manual UWES, psicometricamente os resultados da CFA demonstram melhor ajuste, devendo então ser considerado como um construto trifatorial. Por fim, ainda que de forma prática se possa utilizar o instrumento como unidimensional, resultados da análise LCS (Latent Change Score Model) apontam também para a necessidade de analisar o construto de forma trifatorial, pois não só possuem médias diferentes, mas também variações diferentes ao longo do tempo - algo não capturado de forma unifatorial.

Em suma, a versão brasileira do UWES, de PortoMartins e Benevides-Pereira (2008), mostrou-se adequada para a presente amostra, tanto no grupo Total como no teste/reteste. Ou seja, denota-se que essa versão do instrumento apresentou qualidade tanto para uma única aplicação (Grupo Total) como quando analisado ao longo do tempo. Considera-se, assim, que o objetivo do presente estudo foi atingido.

Sendo o estudo da saúde ocupacional um fator importante até mesmo para a compreensão da saúde de forma geral, um aspecto que caracteriza um estímulo à pesquisa do engajamento no trabalho é o fato de estar associado a variáveis como performance, satisfação e recursos laborais e existir um número reduzido de publicações sobre o tema no Brasil.

Como limitações deste estudo destaca-se o fato da realização em somente uma categoria profissional, assim como a coleta de dados ter ocorrido em uma única empresa, prejudicando a generalização dos resultados. Sugere-se, então, a ampliação de estudos de validação dessa versão do instrumento com amostras diversificadas e em regiões diferentes, uma vez que o Brasil pode ser considerado um país multicultural e continental. Ainda, podem ser realizados testes com mais de duas coletas que possibilitem não só testar modelos LCS (Latent Change Score Model), mas também modelos LGM (Latente Growth Model) para verificar que tipo de variação existe no tempo (linear, quadrática etc.) e também, com amostras mais heterogêneas, que possibilitam uma comparação dos resultados entre diversos grupos.

Por fim, a avaliação do engajamento por meio do UWES também se mostra relevante e pode ser utilizada como indicador estratégico às organizações, sendo uma relevante ferramenta de gestão no sentido de identificar indicadores positivos relacionados à saúde ocupacional e contribuir com o processo de tomada de decisão dentro das organizações.

\section{Agradecimentos}

Não há menções.

\section{Financiamento}

A presente pesquisa não recebeu nenhuma fonte de financiamento sendo custeada com recursos dos próprios autores.

\section{Contribuições dos autores}

Todos os autores contribuíram substancialmente para a elaboração do delineamento da pesquisa, análise e interpretação dos dados, bem como, para a revisão textual e aprovação da versão final deste estudo. Todos os autores assumem responsabilidade pública pelo conteúdo do manuscrito.

\section{Disponibilidade dos dados e materiais}

Todos os dados e sintaxes gerados e analisados durante esta pesquisa serão tratados com total sigilo devido às exigências do Comitê de Ética em Pesquisa em Seres Humanos. Porém, o conjunto de dados e sintaxes que apoiam as conclusões deste artigo estão disponíveis mediante razoável solicitação ao autor principal do estudo.

\section{Conflitos de interesses}

Os autores declaram que não há conflitos de interesses. 


\section{Referências}

Acosta, H., Salanova, M., \& Llorens, S. (2011). ¿Cómo predicen las prácticas organizacionales el engagement en el trabajo en equipo?: El rol de la confianza organizacional. Ciencia y Trabajo, 13(41), 125-134. Recuperado de http://repositori.uji.es/xmlui/bitstream/ handle/10234/43542/51108.pdf?sequence $=1$

Agnst, R., Benevides-Pereira, A. M. T., \& Porto-Martins, P. C. (2009). UWES Utrecht Work Engagement Scale: Escala de Engagement no Trabalho de Utrecht. Recuperado de http://www.wilmarschaufeli.nl/publications/Schaufeli/Test\%20Manuals/Test_manual_UWES_Brazil.pdf

Bakker, A. B., \& Leiter, M. P. (2010). Where to go from here: Integration and future research on work engagement. Em A. B. Bakker \& M. P. Leiter (Orgs.). Work engagement: A handbook of essential theory and research (pp.181-196). New York: Psychology Press.

Brown, T. A. (2006). Confirmatory factor analysis for applied research. New York: Guilford Press.

Byrne, B. M. (2010). Structural equation modeling with Amos: Basic concepts, applications, and programming. New York: Routledge Taylor \& Francis.

Caldas, C. B., Somensari, P., Costa, S. N., \& Siqueira, M. M. M. (2013). Satisfação e engajamento no trabalho: Docentes temáticos e auxiliares da EAD de universidade privada brasileira. Revista Interinstitucional de Psicologia, 6(2), 225-237. Recuperado de http://pepsic. bvsalud.org/scielo.php?script $=$ sci_arttext\&pid $=$ S1983-82202013000200006

Cavalcanti, M. M. (2013). Engajamento no trabalho, bem-estar no trabalho e capital psicológico: um estudo com profissionais da área de gestão de pessoas (Dissertação de mestrado). Universidade Metodista de São Paulo, São Paulo. Recuperado de http://tede.metodista.br/jspui/bitstream/ tede/40/1/MarcileideMC.pdf

Cavalcanti, M. M., Siqueira, M. M. M., \& Kuniyoshi, M. S. (2015). Engajamento, bem-estar no trabalho e capital psicológico: Um estudo com profissionais da área de gestão de pessoas. Pensamento \& Realidade, 29(4), 42-64.

Cheung, G. W., \& Rensvold, R. B. (2002). Evaluating goodness-of-fit indexes for testing measurement invariance. Structural Equation Modeling, 9, 233-255, 2002.doi: 10.1207/S15328007SEM0902_5

Chungkham, H. S., Ingre, M., Karasek, R., Westerlund, H., \& Theorell, T. (2013). Factor structure and longitudinal measurement invariance of the demand control support model: An evidence from the swedish longitudinal occupational survey of health (SLOSH). Plos/One, 8(8), e70541. doi: 10.1371/journal.pone.0070541.

Dalanhol, N. S., Freitas, C. P. P., Machado, W. L., Hutz, C. S., \& Vazquez, A. C. (2017). Engajamento no trabalho, saúde mental e personalidade em oficiais de justiça. Psico, 48(2), 109-119. doi: 10.15448/1980-8623.2017.2.25885

Damásio, B. F. (2013). Contribuições da Análise Fatorial Confirmatória Multigrupo (AFCMG) na avaliação de invariância de instrumentos psicométricos. PSICO-USF, 15(2), 211-220.

Mattos, C. A. C., Andrade, L., \& Tayse, L. (2019). Engajamento no trabalho: Uma aplicação da análise de agrupamentos entre trabalhadores de um banco privado. Revista FSA, 16(1), 54-74.

Duncan, T. E., \& Duncan, S. C. (2009) The ABC's of LGM: An Introductory Guide to Latent Variable Growth Curve Modeling. Social Personality Psychology Compass, 3(6), 979-991. doi: 10.1111/j.1751-9004.2009.00224.x

Ferreira, M., Valentini, F., Damásio, B., Mourão, L., Porto, J., Chinelato, R., Novaes, V. P., \& Pereira, M. M. (2016). Evidências adicionais de validade da UWES-9 em amostras brasileiras. Estudos de Psicologia, 21(4), 435-445. doi: 10.5935/1678-4669.20160042

Freitas, L. A. V., \& Charão-Brito, L. (2017). Engajamento no trabalho: Um estudo em profissionais multidisciplinares de saúde em um município da região sul. Revista Cesumar - Ciências Humanas e Sociais Aplicadas, 21(2), 407-419.

Gonsalez, E. G., Lourenção, L. G., Teixeira, P. R., Rottaa, D. S., Gazetta C. E., Beretta, D., \& Pinto, M. H. (2017). Engagement no trabalho em profissionais de programas de aprimoramento profissional em saúde. Cadernos Braileiros de. Terapia Ocupacional, 25(3), 509-517. doi: 10.4322/2526-8910.ctoAO0987

Hair, J. F., Black, W. C., Babin, B. J., \& Anderson, R. E (2010). Multivariate data analysis. Englewood: Prentice Hall.

Harju, L., Hakanen, J., \& Schaufeli, W. B. (2016). Can job crafting reduce boredom and increase work engagement? A three-year crosslagged panel study. Journal of Vocational Behavior, 96, 11-20. doi: 10.1016/j.jvb.2016.07.001

Hjermstad, M. J., Fossa, S. D., Bjordal, K., \& Kaasa, S. (1995). Test/retest study of the European Organization for Research and Treatment of Cancer Core Quality-of-Life Questionnaire. Journal of Clinical Oncology, 13(5), 1249-1254. doi: 10.1200/JCO.1995.13.5.1249

Hooper, D., Coughlan, J., \& Mullen, M.R. (2008). Structural Equation Modelling: Guidelines for determining model fit. Electronic Journal of Business Research Methods, 6(1), 53-60. doi: 10.21427/D7CF7R

Kievit, R. A., Brandmaier, A. M., Ziegler, G., van Harmelen, A-L., de Mooij, S. M. M., Moutoussis, M., Goodyer, I. M., Bullmore, E., Jones, P. B., Fonagy, P., the NSPSN Consortium, Lindenberger, U., \& Dolan, R.J. (2018) Developmental cognitive neuroscience using latent change score models: A tutorial and applications. Developmental Cognitive Neuroscience, 33, 99-117. doi: 10.1016/j.dcn.2017.11.007

Kline, R. B. (2011). Principles and Practice of Structural Equation Modeling/Rex B. Kline, 3rd ed. New York: Guilford Press.

Kulikowski, K. (2017). Do we all agree on how to measure work engagement? Factorial validity of Utrecht Work Engagement Scale as a standard measurement tool: A literature review. International Journal of Occupational Medicine and Environmental Health, 30( 2), $161-175$. doi: 10.13075/ijomeh.1896.00947

Leon, M., \& Halbesleben, J. R. B. (2013). Construindo resiliência para melhorar o bem-estar dos funcionários. Em A. M. Rossi, J. A. Meurs, \& P. L. Perrewé (Orgs.). Stress e qualidade de vida no trabalho (pp. 68-85). São Paulo: Atlas.

Little, T. D. (2013). Longitudinal Structural Equation Modeling (Methodology in the Social Sciences). 1st Edition, Kindle Edition. The Guilford Press.

Little, T. D., Bovaird, J. A., \& Sleger, D. W. (2006) Methods for the analysis of change. Em D. K. Mroczek \& T. D. Little (Org.). Handbook of personality development. Mahwah, NJ, US: Lawrence Erlbaum Associates Publishers.

Lourenção, L. G. (2018). Qualidade de vida, engagement, ansiedade e depressão entre gestores de Unidades da Atenção Primária à Saúde. Revista Portuguesa de Enfermagem de Saúde Mental, 20, 58-64. doi: 10.19131/rpesm.0227

Machado, P. G. B. (2010). Engagement e burnout em motoristas e cobradores de ônibus. VJornada de Saúde Mental e Psicanálise da PUCPR: Curitiba. Recuperado de http://www2.pucpr.br/reol/index.php/JM?dd1=4427\&dd99=view

Machado, P. G. B., Porto-Martins, P. C. \& Amorim, C. (2012). Engagement no trabalho entre profissionais da educação. Intersaberes, 7(13), 193-214. Recuperado de https://www.uninter.com/intersaberes/index.php/revista/article/view/257 
Machado, P. G. B., \& Porto-Martins, P. C. (2013). Condições organizacionais enquanto terceiras variáveis entre burnout e engagement. Diaphora, 13(1), 35-44.

Machado, P. G. B., Porto-Martins, P. C., \& Benevides-Pereira, A. M. T. (2014). Engagement no trabalho em profissionais de uma empresa de transporte coletivo urbano. Psicologia Argumento, 32(79), 175-185. doi: 10.7213/psicol..argum.32.s02.AO16

Machado, P. G. B., \& Porto-Martins, P. C. (2015). Proposta de protocolo: Indicadores de saúde e enfermidade laboral. Trabalho E Educação, 24(2), 281-293.

Magnan, E. S., Vazquez, A. C. S., Pacico, J. C., \& Hutz, C. S. (2016). Normatização da versão Brasileira da Escala Utrecht de Engajamento no Trabalho. Avaliação Psicológica, 15(2), 133-140.

Malhotra, N. K. (2012). Pesquisa de marketing: Uma orientação aplicada. Porto Alegre: Bookman.

Martinsa, V., Costa, L. V., \& Siqueira, M. M. M. (2015). O impacto do comprometimento afetivo e do engajamento no trabalho sobre os comportamentos de cidadania organizacional. Revista de Administração, Contabilidade e Economia da Fundace, 6(2), 1-13. doi: 10.13059/ racef.v6i2.327

Maslach, C., Jackson, S. E., \& Leiter, M. P. (1996). Maslach Burnout Inventory Manual, 3 ed. Palo Alto CA: Consulting Psychology Press.

Maslach, C., \& Leiter, M. P. (2008). Early predictors of job burnout and engagement. Journal of Applied Psychology, 93(3), 498-512.

Mercali, G. D., \& Costa, S. G. (2019). Antecedentes do engajamento no trabalho dos docentes de ensino superior no Brasil. Revista de Administração Mackenzie, 20(1), 1-28. doi: 10.1590/1678-6971/eramg190081

Milfont, T. L., \& Fischer, R. (2010). Testing Measurement Invariance across groups: applications in cross-cultural research. International Journal of Psychological Research, 3(1), 111-121.

Nakagawa, S., Johnson, P. C., \& Schielzeth, H. (2017). The coefficient of determination R2 and intra-class correlation coefficient from generalized linear mixed-effects models revisited and expanded. Journal of The Royal Society Interface, 14(134), 20170213. doi 10.1098/ rsif.2017.0213

Oliveira, L. B., \& Rocha, J. C. (2017) Engajamento no trabalho: Antecedentes individuais e situacionais e sua relação com a intenção de rotatividade. Revista Brasileira de Gestão de Negócios, 19(65), 415-431.

Pimentel, C. E., Gouveia, V. V., \& Fonseca, P. N. (2005). Escala de Identificação com Grupos Alternativos: Construção e comprovação da estrutura fatorial. Psico-USF, 10(2), 121-127.

Pocinho, M., \& Perestrelo, C. X. (2011). Um ensaio sobre burnout, engagement e estratégias de coping na profissão docente. Educação e Pesquisa, 37(3), 513- 528. doi: 10.1590/S1517-97022011000300005

Porto-Martins, P. C., \& Benevides-Pereira, A. M. T. (2008). Work \& Well-being Survey (UWES) Questionário do Bem-estar e Trabalho. Recuperado de http:/www.wilmarschaufeli.nl/publications/Schaufeli/Tests/UWES_BRA_17.pdf

Porto-Martins, P. C., Machado, P. G. B., \& Benevides-Pereira, A. M. T. (2013). Engagement no trabalho: uma discussão teórica. Fractal, 25(3), 629-643.

Putnick, D. L., \& Bornstein, M. H. (2016). Measurement invariance conventions and reporting: the state of the art and future directions for psychological research. Developmental Review, 41(1), p.71-90.

Ramalho, N., Teles, H., Ramalho, V., \& Ribeiro, S. (2017). Adaptação e validação da Utrecht Work Engagement Scale (UWES) aplicada a assistentes sociais em Portugal. Revista Portuguesa de Investigação Comportamental e Social, 3(2), 10-20.

Rechenberg, L., Goulart, B. N. G., \& Roithmann, R. (2011). Impacto da atividade laboral de teleatendimento em sintomas e queixas vocais - estudo analítico. Jornal da Sociedade Brasileira de Fonoaudiologia, 23(4), 301-307.

Rêgo, S. C. S. (2008). Sofrimento em burnout nos operadores de contact center de uma empresa em Salvador (Dissertação de mestrado). Universidade Federal da Bahia, Salvador, Brasil). Recuperado de https://repositorio.ufba.br/ri/bitstream/ri/7800/1/bbbbb123.pdf

Renosto, A., Biz, P., Hennington, E. A., \& Pattussi, M. P. (2009). Confiabilidade teste-reteste do Índice de Capacidade para o Trabalho em trabalhadores metalúrgicos do sul do Brasil. Revista Brasileira de Epidemiologia, 12(12), 217-225.

Salanova, M., \& Schaufeli, W. B. (2009). El engagement en el trabajo. Madrid: Alianza Editorial.

Salanova, M., Schaufeli, W. B., Llorens, S., Peiro, J. M., \& Grau, R. (2000). Desde el 'burnout' al 'engagement': ¿Una nueva perspectiva? [From 'burnout' to 'engagement': A new perspective?] Revista de Psicología del Trabajo y las Organizaciones, 16, 117-134.

Schaufeli, W. B. (2012). Work Engagement: What do we know and where do we go? Romanian Journal of Applied Psychology, 14(1), 3-10.

Schaufeli W. B. (2015). Engaging leadership in the job demands-resources model. Career Development International, 20(5), p. 446-463. doi: 10.1108/CDI-02-2015-0025

Schaufeli, W. B. (2016). Heavy work investment, personality and organizational climate. Journal of Managerial Psychology, 31, 1057-1073. doi: 10.1108/JMP-07-2015-0259

Schaufeli, W. B. (2017). The Job Demands-Resources model: A 'how to' guide to increase work engagement and prevent burnout. Organizational Dynamics, 46, 120-132. doi: 10.1016/j.orgdyn.2017.04.008

Schaufeli, W. B., \& Bakker, A. (2003). Utrecht Work Engagement Scale (UWES). Occupational Health Psychology Unit: Utrecht.

Schaufeli, W. B., \& Bakker, A. B. (2010). The conceptualization and measurement of work engagement. In A.B. Bakker \& M.P. Leiter (Eds.), Work engagement: A handbook of essential theory and research (pp. 10-24). New York: Psychology Press.

Schaufeli, W. B., Martínez, I. M., Marques-Pinto, A., Salanova, M., \& Bakker, A. B. (2002). Burnout and engagement in university students: A cross-national study. Journal of Cross-Cultural Psychology, 33, 464-481.

Silva, K. S., \& Coutinho. E. S. F. (2005). Escala de apoio social aplicada a uma população de gestantes: confiabilidade teste-reteste e estrutura de concordância dos itens. Cadernos de Saúde Pública, 21(3), 979-983.

Siqueira, M. M. M., Martins, M. C. F., Lima, L. G., Agapito, P. R., \& Souza, W. S. (2011). Construção e validação fatorial da Escala de Engajamento no Trabalho - EEGT. Anais do Congresso Luso-Brasileiro de Psicologia da Saúde e Congresso Ibero-Americano de Psicologia da Saúde, 2/1. Recuperado de http://www.metodista.br/ev/psicologia-da-saude/anais/simposio.pdf/view

Teixeira, P. R., Lourenção, L. G., Gazetta, C. E., Gonsalez, E. G., Rotta, D. S., Pinto, M. H., \& Beretta, D. (2017). Engagement no trabalho em residentes médicos de pediatria. Revista Brasileira de Educação Médica, 41(1), 126-133. doi: 10.1590/1981-52712015v41n1rb20160039

Vazquez, A., Magnan, E., Pacio, J., Hutz, C., \& Schaufeli, W. (2015). Adaptation and Validation of the Brazilian Version of the Utrecht Work Engagement Scale. Psico-USF, 20(2), 207-217. doi: 10.1590/1413-82712015200202 
Verdan, M. G. E., \& Oort, F. J. (2014). Measurement bias detection with Kronecker product restricted models for multivariate longitudinal data: An illustration with health-related quality of life data from thirteen measurement occasions. Frontiers in Psychology: Methods Article, 23, 188-195 doi: 10.3389/fpsyg.2014.01022

Yulita, Dollard, M. F., \& Idris, M. A. (2017). Climate congruence: How espoused psychosocial safety climate and enacted managerial support affect emotional exhaustion and work engagement. Safety Science, 96, 132-142. doi: 10.1016/j.ssci.2017.03.023

\section{Sobre os autores}

Paulo Cesar Porto Martins é psicólogo, doutor em psicologia pela Universidad Autónoma de Madrid (UAM - ESpanha). Atualmente, é professor do curso de administração da PUCPR e responsável pela educação empreendedora da mesma universidade.

Pedro Guilherme Basso Machado é psicólogo (PUCPR), doutor em Psicologia pela Universidad Autónoma de Madrid (UAM Espanha) e Pós-doutorando em Educação pela PUCPR. Atualmente é Professor do curso de Psicologia no Unibrasil.

Juan José Camou Viacava é professor (PUCPR), doutor em administração (ênfase em Marketing e Comportamento do Consumidor) pela Universidade Federal do Paraná (UFPR). Atualmente é professor colaborador do Programa de Pós-Graduação em Administração (Stricto Sensu - PPAD), da Escola de Negócios da Pontifícia Universidade Católica do Paraná (PUCPR).

\section{Como citar este artigo}

Paulo-Martins et al. (2020). Engajamento no Trabalho: Características Psicométricas e Invariância Temporal do UWES. Avaliação Psicológica, 19(3), 277-288. http://dx.doi.org/10.15689/ap.2020.1903.15631.06 